\title{
Treatment of Chronic Spontaneous Urticaria With Benralizumab: Report of Primary Endpoint Per Protocol Analysis, and Exploratory Endpoints
}

\author{
Jonathan Bernstein ${ }^{1}$, Umesh Singh ${ }^{2}$, Marepalli Rao ${ }^{2}$, Karen Berendts ${ }^{3}$, Xiang Zhang ${ }^{2}$, and \\ Diya Mutasim ${ }^{2}$ \\ ${ }^{1}$ University of Cincinnati \\ ${ }^{2}$ University of Cincinnati College of Medicine \\ ${ }^{3}$ Bernstein Clinical Research Center
}

October 27, 2020

\section{Treatment of Chronic Spontaneous Urticaria With Benralizumab: Report of Primary End- point Per Protocol Analysis, and Exploratory Endpoints}

Standard treatments for chronic spontaneous urticaria (CSU) including the second-generation H1antihistamines (SGAH) are often ineffective even with four-times the FDA-recommended dose. ${ }^{1,2}$ Eosinophilic infiltrates and an abundance of interleukin-5 (IL5) in CSU lesions (hives) support a role for IL5 in the pathomechanism of CSU. ${ }^{3}$ Thus, the use of biologic therapies, e.g. benralizumab targeting IL5-receptor- $\alpha$, in treating SGAH-resistant CSU was hypothesized.

A repeated-measures, 24-week study was designed and conducted at an urticaria clinic to determine clinical efficacy of benralizumab in CSU. Twelve SGAH-unresponsive CSU patients (3 males, 9 females; 2 blacks, 10 whites; between ages 32-65 years) having a median daily Urticaria Activity Score (UAS7) ${ }^{4}$ of 4, and pruritus severity [?]2 were enrolled. After a baseline run-in period, subjects were treated with a subcutaneous placebo dose followed by benralizumab 30mg subcutaneously every month $(\times 3$ doses $)$ followed by two offmedication monthly-visits. Subject-reported responses to UAS7 and CU-QoL questionnaires were recorded at the monthly visits. The primary and exploratory endpoints were the change in UAS7 and Chronic Urticaria Quality-of-Life Total Score (CUQoLTS) respectively, from 4 weeks after placebo dose (visit 2) to 4 weeks after last dose of benralizumab (visit 5). Nine subjects completed the study; three withdrew after the first benralizumab dose. An intent-to-treat (ITT) analysis $(n=12)$ of the primary endpoint has been reported previously. ${ }^{5}$ Per-protocol $(\mathrm{PP})$ analysis $(\mathrm{n}=9)$ of the primary endpoint, and PP vs. ITT of the exploratory endpoint are reported here. It was presumed that lesions were not self-limiting, and any improvement in outcomes during the study were because of intervention. Non-responders to benralizumab were identified if at any time during the 16 weeks after the first benralizumab dose there was $<40 \%$ improvement in UAS7 from baseline vs. responders if UAS7 was [?]6.

The average duration of urticarial symptoms was 7.0 years. Baseline UAS7 and CUQoLTS ranged between 22-42 and 36-95 respectively. Both outcomes significantly improved at visit 5 vs. visit 2 in 7 of $9(78 \%)$ subjects completing the study. The average difference (95\% CL) between visit 2 and visit 5 for UAS7, was $-15.5(-4.1,-26.8, \mathrm{p}=0.003)$ and for CUQoLTS, using ITT analysis, was-13.2 $(-2.4,-24.0, \mathrm{p}=0.0005)$ or, using PP analysis, was -11.6 (-0.8, -22.4, p=0.03) (Figure-1 ). Five responders reported no hives/pruritus $(\mathrm{UAS} 7=0)$ at visit 5 or 6.

Between responders and non-responders, the average age (51.6 vs. $53, \mathrm{p}=0.9)$ and symptom duration ( 5 
vs. 9 years, $\mathrm{p}=0.5)$ did not differ significantly. However, the adjusted mean UAS7 percentage difference, adjusted for symptom duration, age, and blood eosinophils (eos\%), from visit 2 till visit 5 was $-84 \%$ for responders and $+7.5 \%$ for non-responders, $\mathrm{p}=0.0009$ (Table-1). The average baseline UAS7 was not statistically significant ( 27 vs. $37.5, \mathrm{p}=0.5$ ) between groups however, the baseline mean difference for eos\% was $-6+-1(\mathrm{p}=0.001)$, and for basophil\% was $-1.2+-0.2, \mathrm{p}=0.02)$, which were not observed at visit 5 (eos\%: $0+-1, \mathrm{p}=0.9$; basophil\%: $0.2+-0.2, \mathrm{p}=0.8)($ Table-1 $)$. Thus, clinical improvement among responders was independent of baseline disease severity. Non-responsiveness to benralizumab, measured by changes in UAS7, are likely due to other mechanistic factors unrelated to eos\% which are eliminated by blocking IL-5R with benralizumab(S-Figure-1) .

UAS7 and CU-QoLTS values were significantly correlated $\left(\mathrm{r}^{2}=0.9, \mathrm{p}<0.0001\right)$ (S-Table-3) . CU-QoL components that improved significantly were the pruritus/wheal scores, urticarial interference with physical activities, sleep and spare time (S-Table-4) .

This study supports the use of benralizumab for treatment of SGAH-unresponsive CSU. Benralizumabrelated improvements in UAS7 and CUQoLTS reported here are similar to the efficacy of omalizumab in CSU reported in a previous study (S-Table-5) . ${ }^{6}$ The sustained significant improvement in urticarial lesions based on subject-reported outcomes by benralizumab warrants further investigation of underlying biologic pathways to better elucidate the role of IL-5 in CSU.

\section{Figures and Tables}

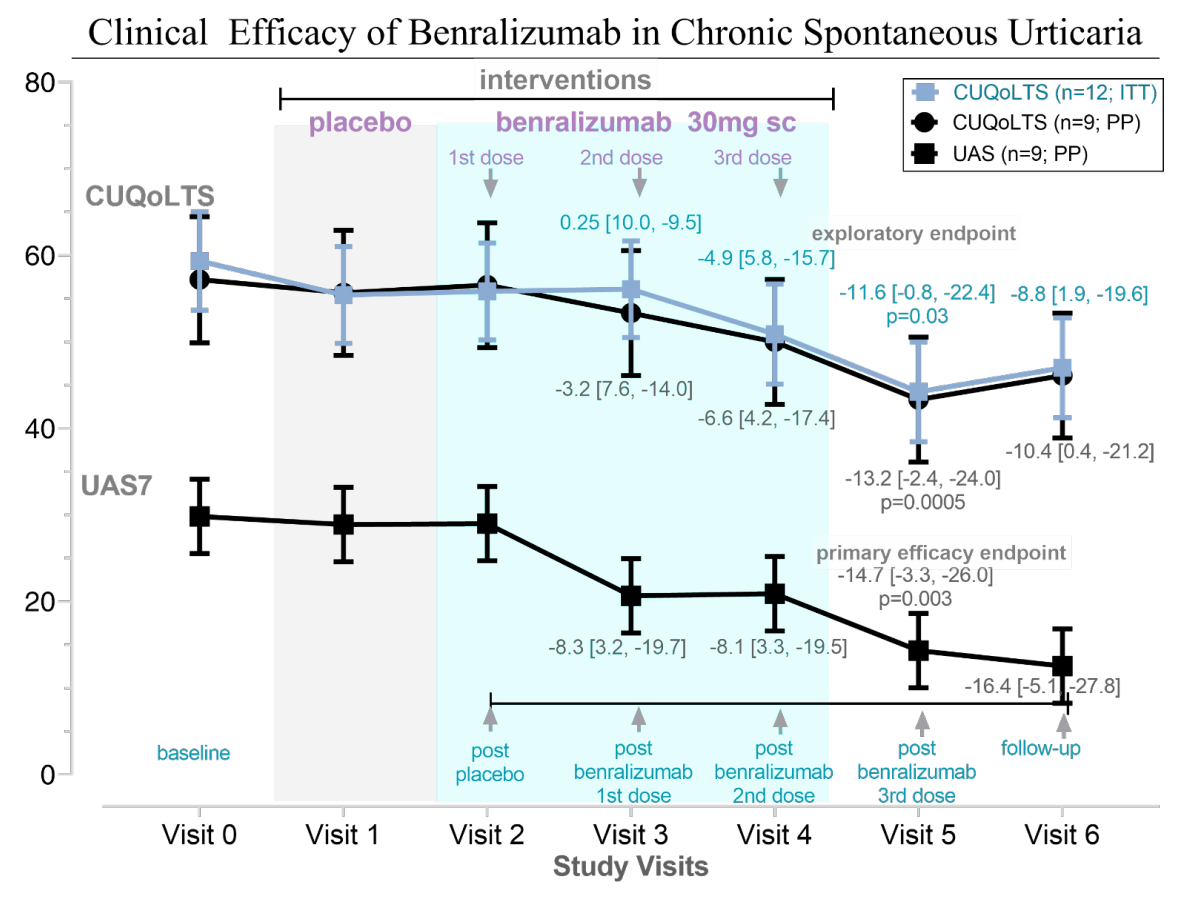

Figure 1. Mean UAS7 and CUQoLTS at each visit during the study. Mean Difference (95\%CI) from the post-placebo visit (visit 2) to the respective visits are shown.

Table-1. Differences between Benralizumab responders (either complete i.e., UAS7 $=0$ at visit 5 , or partial i.e., UAS7[?]6 at visit $5, \mathrm{n}=7)$ versus non-responders $(\mathrm{n}=2)$ using per protocol analysis (i.e., subjects completing the study, $\mathrm{n}=9$ ).

\begin{tabular}{lll}
\hline Parameter & Parameter & Responder \\
\hline Average Symptom Duration & Average Symptom Duration & 5.1
\end{tabular}




\begin{tabular}{lll}
\hline Parameter & Parameter & Responder \\
\hline Average Age of Subjects & Average Age of Subjects & 51.6 \\
Average UAS7(Visit 2) \pm SE & Average UAS7(Visit 2) \pm SE & $26 \pm 3$ \\
Average UAS7(Visit 5) \pm SE & Average UAS7(Visit 5) \pm SE & $7 \pm 3$ \\
Average $\Delta$ UAS7(Visit 5 - Visit 2) & Average $\Delta$ UAS7(Visit 5 - Visit 2) & $-84 \%$ \\
Parameter & Comparison Group-Visit & Comparison Group-Visit \\
UAS7 & (Responder-Visit 1) vs. (Non-Responder-Visit 1) & (Responder-Visit 1) vs. (Non- \\
& (Responder-Visit 5) vs. (Non-Responder-Visit 5) & (Responder-Visit 5) vs. (Non- \\
Blood Neutrophil \% & (Responder-Visit 1) vs. (Non-Responder-Visit 1) & (Responder-Visit 1) vs. (Non- \\
& (Responder-Visit 5) vs. (Non-Responder-Visit 5) & (Responder-Visit 5) vs. (Non- \\
Blood Eosinophil \% & (Responder-Visit 1) vs. (Non-Responder-Visit 1) & (Responder-Visit 1) vs. (Non- \\
& (Responder-Visit 5) vs. (Non-Responder-Visit 5) & (Responder-Visit 5) vs. (Non- \\
& (Non-Responder-Visit 1) vs. (Non-Responder-Visit 5) & (Non-Responder-Visit 1) vs. ( \\
& (Responder-Visit 1) vs. (Responder-Visit 5) & (Responder-Visit 1) vs. (Resp \\
& (Responder-Visit 1) vs. (Non-Responder-Visit 1) & (Responder-Visit 1) vs. (Non- \\
Blood Lymphocyte \% & (Responder-Visit 5) vs. (Non-Responder-Visit 5) & (Responder-Visit 5) vs. (Non- \\
& (Non-Responder-Visit 1) vs. (Non-Responder-Visit 5) & (Non-Responder-Visit 1) vs. ( \\
& (Responder-Visit 1) vs. (Responder-Visit 5) & (Responder-Visit 1) vs. (Resp \\
& (Responder-Visit 1) vs. (Non-Responder-Visit 1) & (Responder-Visit 1) vs. (Non- \\
& (Responder-Visit 5) vs. (Non-Responder-Visit 5) & (Responder-Visit 5) vs. (Non- \\
Blood Basophil \% & (Non-Responder-Visit 1) vs. (Non-Responder-Visit 5) & (Non-Responder-Visit 1) vs. ( \\
& (Responder-Visit 1) vs. (Responder-Visit 5) & (Responder-Visit 1) vs. (Resp \\
& &
\end{tabular}

* determined by regression analysis adjusted for symptom duration, subject age, and eos\% at baseline.

\section{References}

1. Amin P, Levin L, Holmes SJ, Picard J, Bernstein JA. Investigation of patient-specific characteristics associated with treatment outcomes for chronic urticaria. The journal of allergy and clinical immunology In practice. 2015;3(3):400-407.

2. Zuberbier T, Aberer W, Asero R, et al. The EAACI/GA(2)LEN/EDF/WAO guideline for the definition, classification, diagnosis and management of urticaria. Allergy. 2018;73(7):1393-1414.

3. Caproni M, Giomi B, Volpi W, et al. Chronic idiopathic urticaria: infiltrating cells and related cytokines in autologous serum-induced wheals. Clin Immunol. 2005;114(3):284-292.

4. Hawro T, Ohanyan T, Schoepke N, et al. The Urticaria Activity Score-Validity, Reliability, and Responsiveness. The journal of allergy and clinical immunology In practice. 2018;6(4):1185-1190 e1181.

5. Bernstein JA, Singh U, Rao MB, Berendts K, Zhang X, Mutasim D. Benralizumab for Chronic Spontaneous Urticaria. N Engl J Med.2020;383(14):1389-1391.

6. Kaplan AP, Joseph K, Maykut RJ, Geba GP, Zeldin RK. Treatment of chronic autoimmune urticaria with omalizumab. J Allergy Clin Immunol. 2008;122(3):569-573.

\section{Author List}

Jonathan A. Bernstein, M.D. ${ }^{1}$

Umesh Singh, M.D., Ph.D. ${ }^{1}$

Marepalli B. Rao, Ph.D. ${ }^{1}$ Karen Berendts, B.S., R.R.T. ${ }^{2}$ Xiang Zhang, Ph.D. ${ }^{1}$ Diya Mutasim, M.D. ${ }^{1}$

${ }^{1}$ University of Cincinnati College of Medicine, Cincinnati, $\mathrm{OH}^{2}$ Bernstein Clinical Research Center, Cincinnati, $\mathrm{OH}$ 


\section{Corresponding Author}

Jonathan A Bernstein, MD

Professor of Medicine

University of Cincinnati College of Medicine

Department of Internal Medicine

Division of Immunology, Allergy Section

MSB 7413, 231 Albert Sabin Way, Cincinnati OH 45267-0563

Tel: (513)558-5533

Fax: (513)558-3799

E-mail: jonathan.bernstein@uc.edu

\section{Funding}

Supported by AstraZeneca

\section{Conflicts of Interest}

Dr. Bernstein reports grants and personal fees from Astra Zeneca, during the conduct of the study; grants and personal fees from Shire/Takeda, grants and personal fees from CSL Behring, grants and personal fees from Pharming, grants and personal fees from Biocryst, grants and personal fees from Kalvista, grants and personal fees from Ionis, grants and personal fees from Novartis, grants and personal fees from Genentech, grants and personal fees from Sanofi-Regeneron, grants and personal fees from Allakos, outside the submitted work, and reports being a member of the Joint Task Force for the AAAAI and ACAAI, a member of the CU UCARE guideline committee and a UCARE center of excellence, and a member of the MAB of the HAEA organization.

All other authors have nothing to disclose. 